\title{
Paroxysmal compulsion to handle keys in a computer operator due to meningioma in the left supplementary motor area
}

\author{
H. Tei ${ }^{\mathrm{a}, \mathrm{b}, *}$, M. Iwata ${ }^{\mathrm{a}}$ and Y. Miura ${ }^{\mathrm{a}}$ \\ a Department of Neurology, Neurological Institute, \\ Tokyo Women's Medical College, Tokyo, Japan \\ ${ }^{\mathrm{b}}$ Department of Neurology, Toda Central General \\ Hospital, Saitama, Japan
}

\begin{abstract}
We describe the case of a computer operator who experienced paroxysmal attacks several times in which she felt a compulsion to handle keys with her right hand or actually her right hand moved involuntarily in a key-handling rhythm. Cranial CT and MRI revealed a mass lesion in the left medial aspect of the frontal lobe (supplementary motor area). After the removal of this tumor (meningioma), there were no more paroxysmal attacks. We suggest that voluntary movements controlled by the supplementary motor area were deranged by seizures provoked by the tumor. This case is attractive in relation to obsessive-compulsive disorder.
\end{abstract}

Keywords: Supplementary motor area, brain tumor, motor control, seizure, obsessive-compulsive disorder

\section{Introduction}

Recent studies have suggested that the supplementary motor area plays a major role in the initiation and control of voluntary movements, especially in complex ones $[3,9,10,11]$. We describe the case of a computer operator with a meningioma in the left supplementary motor area whose first clinical manifestation was paroxysmal compulsion to handle keys with her right hand. This case is also attractive in relation to obsessive-compulsive disorder.

${ }^{*}$ Corresponding author: H. Tei, Department of Neurology, Toda Central General Hospital 1-19-3, Hon-cho, Toda City, Saitama Prefecture, 335 Japan. Tel.: +81 48442 1111; Fax: +81 484430104 .

\section{Case report}

The patient was a 35-year-old woman who had been working as a computer operator for several years. There was no past medical history. She was right handed and all her relatives were right-handed. One evening, after overworking for several days (operating a computer for more than 10 hours a day), she was writing a report with a pen at her desk. After she put the pen down on the desk, she felt a powerful compulsion to operate a computer with her right hand. Immediately, she held her right hand with her left hand. This compulsion disappeared within two minutes. Similar events occurred three times within six months. One afternoon about a year later, she met a friend and talked with her. When the conversation stopped for a minute, she felt a powerful compulsion to operate a computer with her right hand for a moment, then actually started moving involuntarily in a key-handling rhythm for a few seconds. She held her right hand down immediately with her left hand. Then she intended to explain this phenomenon to her friend, but she could not speak at all. This involuntary right hand movement and speech arrest resolved within a few minutes without any disturbance of consciousness. In the following week, she experienced another similar attack. This time,instead of speech arrest, continuous vocalization of ' $\mathrm{zu}, \mathrm{zu}, \mathrm{zu}, \mathrm{zu}, \ldots$. ', occurred for a minute. She visited the Department of Neurology at Tokyo Women's Medical College.

General physical examination revealed no abnormal findings. Neurological examination revealed no abnormal findings, in cranial nerves, motor system, deep tendon reflexes, sensory system, coordination, standing or gait. No pathological reflexes, including grasp reflex, were elicited. On neuropsychological examination, she was attentive, cooperative and well orien- 


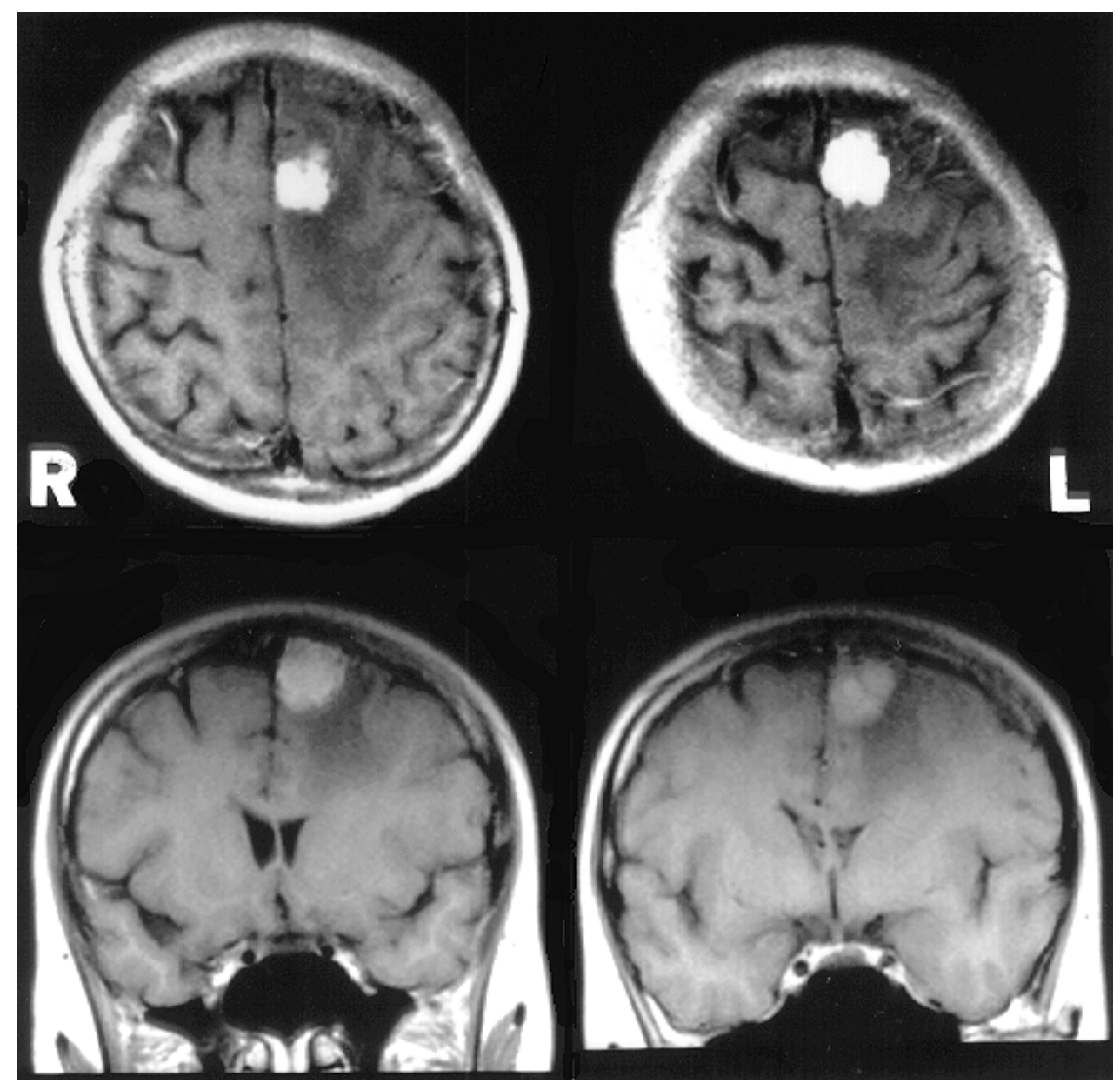

Fig. 1. Gadolinium-enhanced $T_{1}$-weighted MRI images revealed an enhanced mass lesion in the left medial frontal lobe with surrounding low intensity areas.

tated to time, place and person. There was no aphasia, apraxia nor agnosia. Calculation, right-left discrimination and finger gnosia were intact. Her WAIS score was as follows: verbal IQ $=93$, performance $\mathrm{IQ}=84$, full scale IQ $=88$. We asked her to perform bi-manual movements such as cut a piece of paper with scissors, put a letter into an envelope, etc. She could carry out these movements without any clumsiness. She was able to perform Luria's sequential movements [7] skillfully with both hands. Alien hand signs, diagonistic dyspraxia and compulsive manipulation of tools, were not observed. None of the classic callosal disconnection syndromes such as left unilateral agraphia, tactile anomia nor ideomotor apraxia were detected.

Interictal electroencephalography was normal. Cranial CT and MRI (Fig. 1) showed a mass lesion in the left medial frontal lobe.
Enhanced lesion on MRI (gadolinium-enhanced $T_{1}$ weighted image, Fig. 1)was restricted in the medial aspect of Brodmann's area 6, i.e., the supplementary motor area (according to the template of [2]).

Total removal of the tumor was performed. Pathologically, the tumor was meningothelial meningioma with hemangioblastic component. Although she felt slight weakness in the right leg for several days after the operation, she was discharged from our hospital without any residual symptoms. She was treated with sodium valproate and there have been no paroxysmal attacks for two years.

\section{Discussion}

While the interictal EEG was normal, the paroxysmal compulsion to handle keys in our patient was 
probably caused by epilepsy provoked by a mass lesion in the left medial aspect of the frontal lobe. It has been suggested that the EEGs especially interictal EEG of frontal lobe epilepsy have relatively poor sensitivity and specificity and are often normal without any epileptiform abnormalities $[5,8,13]$. The clinical manifestations of our patient in which there were brief episodes of right hand sensory (compulsion to handle keys) and later motor abnormality (actually moved in a key-handling rhythm) contralateral to the lesion, and speech arrest without disturbance of consciousness correspond with the characteristics of seizures caused by supplementary motor area foci $[8,13]$.

Recent studies have suggested that the supplementary motor area plays a major role in the initiation and control of voluntary movements, especially in complex ones [3, 9, 10, 11]. Fried et al. [3] performed electrical stimulation on the supplementary motor area of 13 patients with intractable epilepsy and found that subjective-sensory responses without overt motor activity were elicited in nine of them. They described three types of subjective-sensory response, the third type being a subjective 'urge' to perform a movement or the anticipation that a movement was going to occur. At some stimulated sites where such responses were elicited, stimulation at higher current evoked an overt motor response. These results correspond to those of our patient well, in that at first, she merely felt a compulsion to operate a computer with her right hand, but later, her right hand actually moved in a key-handling rhythm. This suggests that enlargement of the tumor changed the character of the seizure. Why she felt an impulse during paroxysmal attacks, or actually moved in the rhythm of operating a computer is not clear. In the nine patients described by Fried et al. [3] mentioned above, no specific subjective-sensory responses like those in our patient were noted. The supplementary motor area may contribute to the establishment of new motor programs and probably controls the execution of established subroutines according to external and internal inputs [9]. In the present patient, the complex movement of typewriting can be recognized as an established subroutine by overtraining. We suggest that the seizure evoked from the left medial frontal meningioma disrupted the above mentioned control system and provoked the established subroutine, i.e., operating a computer.

The symptoms of compulsive urge to operate a computer with inner resistance of our case are also attractive in association with obsessive-compulsive disorder (OCD). OCD is a relatively frequent anxiety dis- order characterized by the presence of intrusive and senseless ideas, thoughts, urges, and images (obsessions), as well as by repetitive cognitive and physical activities that are performed in a ritualistic way (compulsions) $[1,4]$. Current studies indicate that structure damage to the circuits involving the orbitofrontal gyrus, caudate nucleus, and anterior cingulate cortex is implicated in the pathogenesis of both idiopathic OCD and OCD with focal brain lesions, and OCD may occur in association with complex partial seizures originating in the temporal and frontal cortices or in the anterior cingulate gyrus $[1,6,12]$, agreeing with our case. Ward [12] reported three cases with frontal lesions which showed transient feelings of compulsion, probably caused by epilepsy. They are similar to our case, but they experienced feelings of compulsion without actual movement. Ward speculated that planning of voluntary movement mediated by frontal lobe was disordered by epilepsy, but the mechanism for judging overall coherence of behaviour must have been intact, so internal resistance was maintained and action inhibited.

\section{Acknowledgements}

We wish to thank Dr. Toshiki Fujioka of the Fourth Department of Internal Medicine, Toho University Hospital for his supply of the patient's clinical data.

\section{References}

[1] M.L. Berthier, J. Kulisevsky, A. Gironell and J.A. Heras, Obsessive-compulsive disorder associated with brain lesions: clinical phenomenology, cognitive function, and anatomic correlates, Neurology 47 (1996), 353-361.

[2] H. Damasio and A.R. Damasio, Lesion analysis in neuropsychology, Oxford University Press, Oxford, 1989.

[3] I. Fried, A. Katz, G. McCarthy, K.J. Sass, P. Williamson, S.S. Spencer and D.D. Spencer, Functional organization of human supplementary motor cortex studied by electrical stimulation, The Journal of Neuroscience 11 (1991), 3656-3666.

[4] M.A. Jenike, H.C. Breiter, L. Baer, D.N. Kennedy, C.R. Savage, M.J. Olivares, R.L. O'Sullivan, D.M. Shera, S.L. Rauch, N. Keuthen, B.R. Rosen, V.S. Caviness and P.A. Filipek, Cerebral structural abnormalities in obsessive-compulsive disorder. a quantitative morphometric magnetic resonance imaging study, Archives of General Psychiatry 53 (1996), 625-632.

[5] D.T. Laskowitz, M.R. Sperling, J.A. French and M.J. O'Connor, The syndrome of frontal lobe epilepsy: characteristics and surgical management, Neurology 45 (1995), 780787.

[6] B. Levin and M. Duchowny, Childhood obsessive-compulsive disorder and cingulate epilepsy, Biological Psychiatry 30 (1991), 1049-1055. 
[7] A.R. Luria, Higher cortical functions in man, Basic Books, New York, 1966.

[8] H.H. Morris, D.S. Dinner, H. Lüders, E. Wyllie and R. Kramer, Supplementary motor seizures: clinical and electroencephalographic findings, Neurology 38 (1988), 10751082.

[9] J.M. Orgogozo and B. Larsen, Activation of the supplementary motor area during voluntary movement in man suggest it works as a supramotor area, Science 206 (1979), 847-850.

[10] S.M. Rao, J.R. Binder, P.A. Bandettini, T.A. Hammeke, F.Z. Yetkin, A. Jesmanowicz, L.M. Lisk, G.L. Morris, W.M Mueller, L.D. Estkowski, E.C. Wong, V.M. Haughton and J.S.
Hyde, Functional magnetic resonance imaging of complex human movements, Neurology 43 (1993), 2311-2318.

[11] P. Remy, M. Zilbovicius, A. Leroy-Willig, A. Syrota and Y. Samson, Movement-and task-related activations of motor cortical areas: a positron emission tomographic study, Annals of Neurology 36 (1994), 19-26.

[12] C.D. Ward, Transient feelings of compulsion caused by hemispheric lesions: three cases, Journal of Neurology, Neurosurgery, and Psychiatry 51 (1988), 266-268.

[13] K. Waterman, S.J. Purves, B. Kosaka, E. Strauss and J.A. Wada, An epileptic syndrome caused by mesial frontal lobe seizure foci, Neurology 37 (1987), 577-582. 


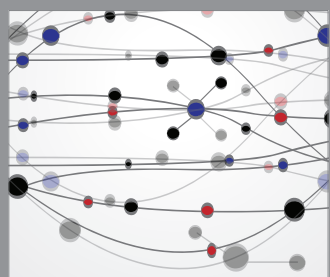

The Scientific World Journal
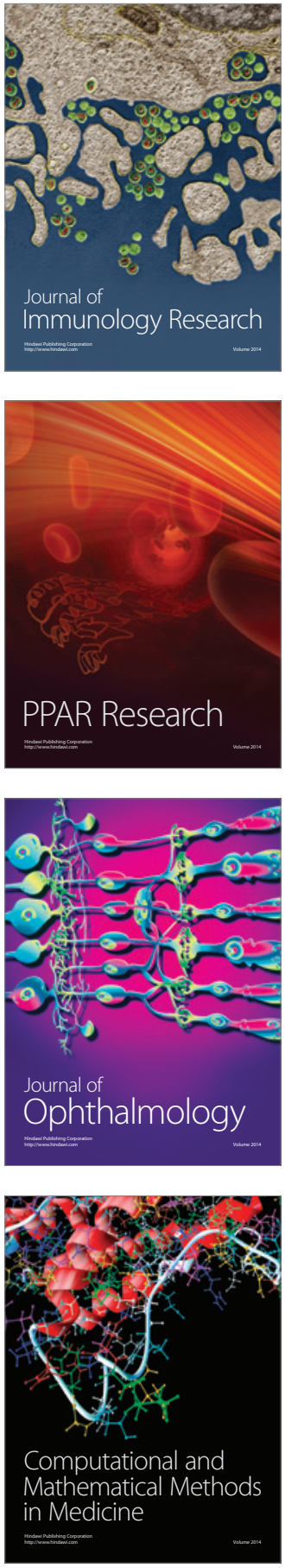

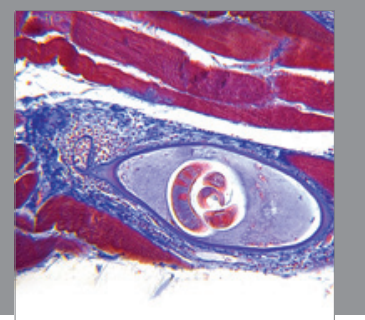

Gastroenterology

Research and Practice
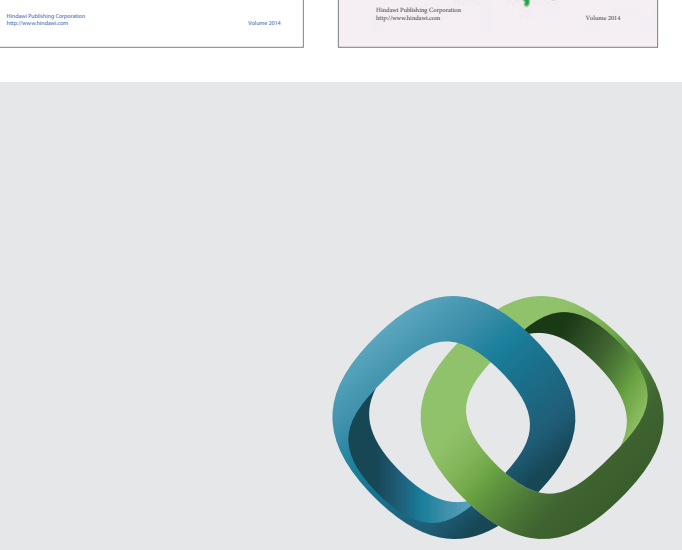

\section{Hindawi}

Submit your manuscripts at

http://www.hindawi.com
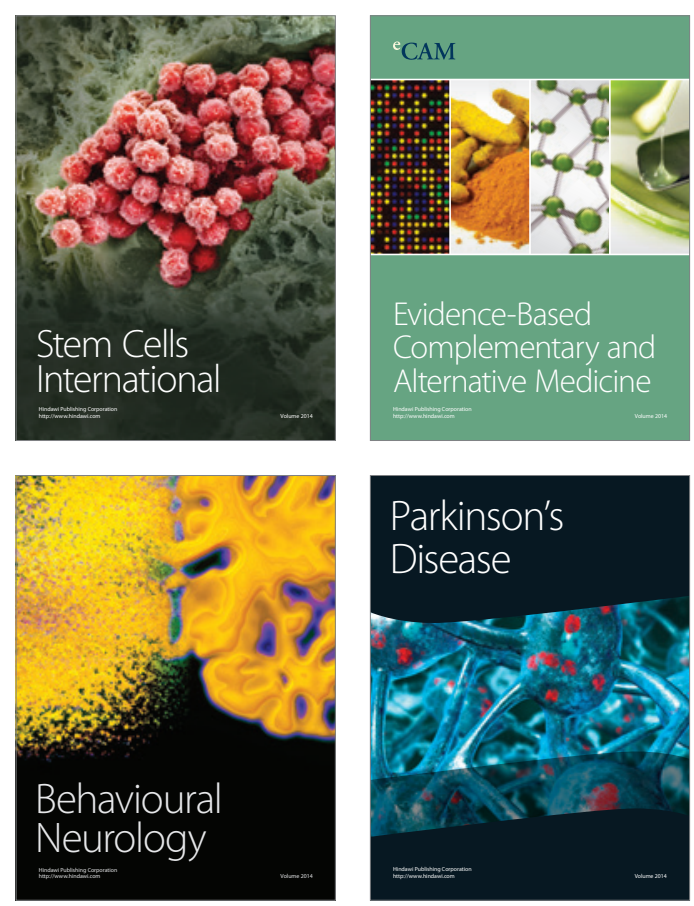

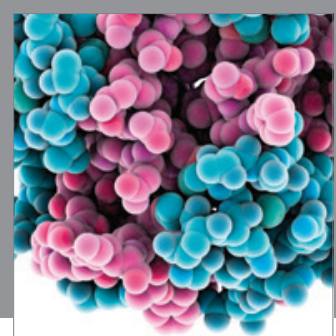

Journal of
Diabetes Research

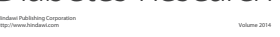

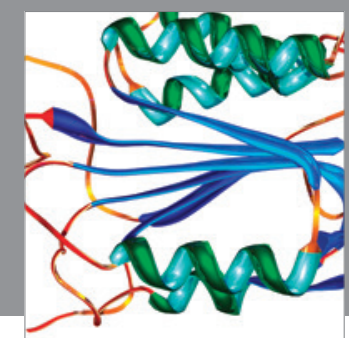

Disease Markers
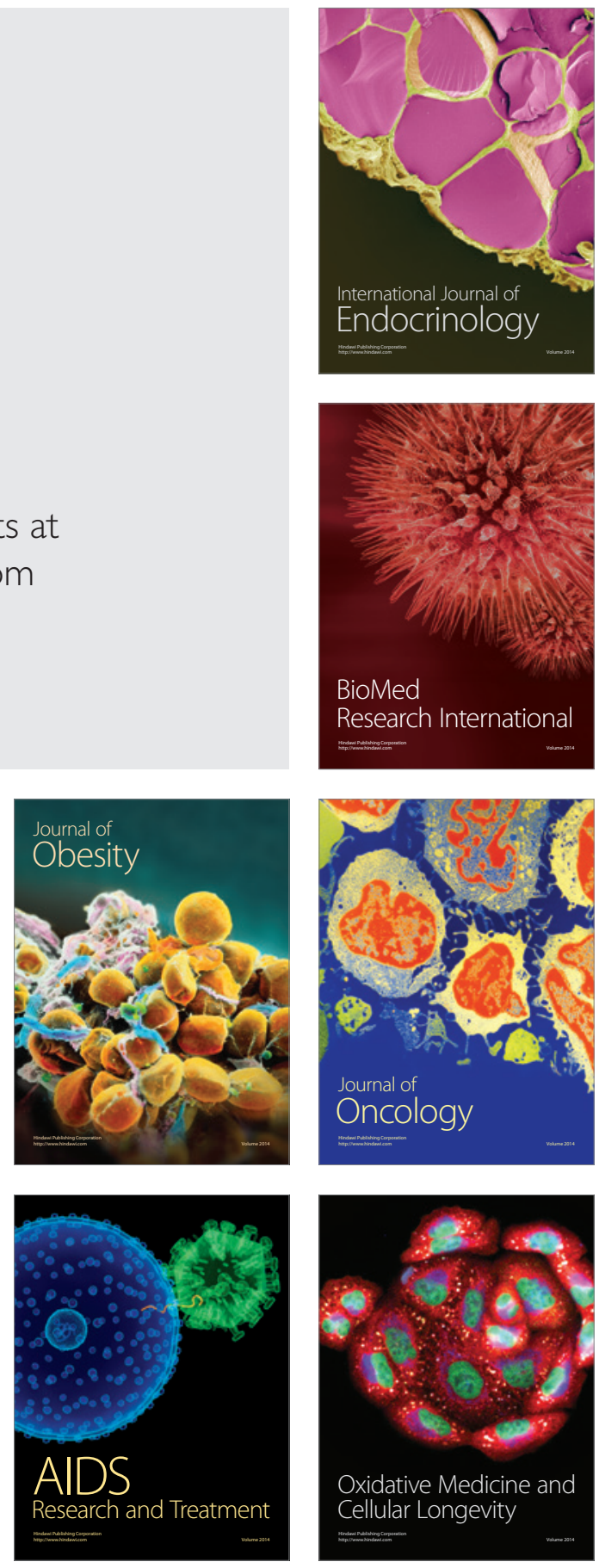